\title{
Noise transmission during the dynamic pattern formation in fly embryos
}

\author{
Zhe Yang ${ }^{1, \dagger}$, Xiaoxuan $\mathrm{Wu}^{1, \dagger}$, Ning Yang ${ }^{1}$ and Feng Liu ${ }^{1,2, *}$ \\ ${ }^{1}$ Center for Quantitative Biology, Peking University, Beijing 100871, China \\ 2 State Key Laboratory of Nuclear Physics and Technology, School of Physics, Peking University, Beijing 100871, China \\ * Correspondence: liufeng-phy@pku.edu.cn
}

Received July 1, 2017; Revised August 16, 2017; Accepted September 15, 2017

\begin{abstract}
Background: Developmental patterning is highly reproducible and accurate at the single-cell level during fly embryogenesis despite the gene expression noise and external perturbations such as the variation of the embryo length, temperature and genes. To reveal the underlying mechanism, it is very important to characterize the noise transmission during the dynamic pattern formation. Two hypotheses have been proposed. The "channel" scenario requires a highly reproducible input and an accurate interpretation by downstream genes. In contrast, the "filter" scenario proposes a noisy input and a noise filter via the cross-regulation of the downstream network. It has been under great debates which scenario the fly embryogenesis follows.

Results: The first 3-h developmental patterning of fly embryos is orchestrated by a hierarchical segmentation gene network, which rewires upon the maternal to zygotic transition. Starting from the highly reproducible maternal gradients, the positional information is refined to the single-cell precision through the highly dynamical evolved zygotic gene expression profiles. Thus the fly embryo development might strictly fit into neither the originally proposed "filter" nor "channel" scenario. The controversy that which scenario the fly embryogenesis follows could be further clarified by combining quantitative measurements and modeling.

Conclusions: Fly embryos have become one of the perfect model systems for quantitative systems biology studies. The underlying mechanism discovered from fly embryogenesis will deepen our understanding of the noise control of the gene network, facilitate searching for more efficient and safer methods for cell programming and reprogramming, and have the great potential for tissue engineering and regenerative medicine.
\end{abstract}

Keywords: pattern formation; gene regulatory network; noise; embryogenesis; Drosophila

\begin{abstract}
Author summary: It is intriguing how the development of organisms is highly reproducible and accurate despite the external and internal noise. The key to solve this mystery is to explore the noise transmission during development and discover the underlying mechanism. The developmental system could generate precise inputs and outputs in each step, or gradually generate a final precise output by filtering a noisy input according to the "channel" or "filter" scenario, respectively. The quantitative studies on the early development of the fruit fly's embryos show that the developmental process is highly dynamic and its noise transmission may employ a hybridized strategy.
\end{abstract}

\section{INTRODUCTION}

The development of complex multicellular organisms from a single zygotic cell is highly dynamical yet amazingly accurate and reproducible [1,2]. For instance, cephalic furrow (CF) is transiently formed during early morphogenesis in fly (Drosophila) embryos [3,4]. The location of $\mathrm{CF}$ is highly reproducible from embryo to embryo (Figure 1A). Its relative position with the respect to the anterior pole of the embryo is $33.8 \pm 1.4 \%$ embryo length (L) (Figure 1B), indicating that the cell fate determination for $\mathrm{CF}$ formation achieves nearly single-

\footnotetext{
${ }^{\dagger}$ These authors contributed equally to this work.
} 
cell precision [5], as the fly embryo consists of about 100 columns of cells along the anterior-posterior (AP) axis, and the standard deviation of the CF location is about $1 \%$ $\mathrm{L}$, corresponding to the width of a single cell. However, quantitative imaging at the single-cell level reveals that gene expression noise is profound and ubiquitous (Figure 1C) [6], and can be divided into intrinsic noise and extrinsic noise (Figure 1D) [7]. Intrinsic noise stems from the stochasticity of diffusional reaction processes. Extrinsic noise originates from the environmental fluctuation such as cellular component changes, and temperature variation. It is intriguing how the gene regulation network orchestrates the highly accurate and reproducible developmental processes at the single-cell level in multicellular organisms despite the intrinsic and extrinsic noise.

A

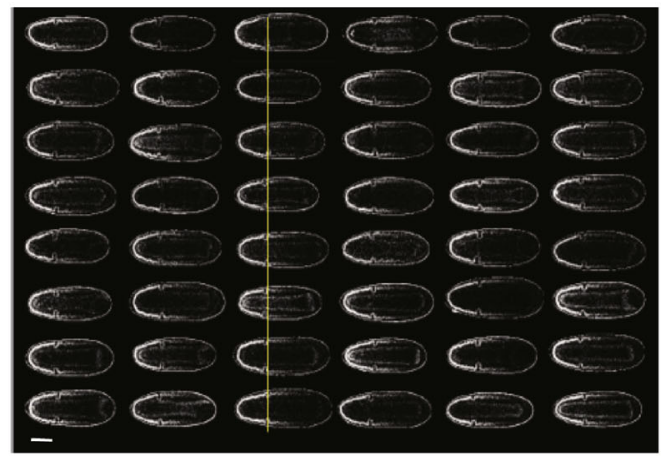

B

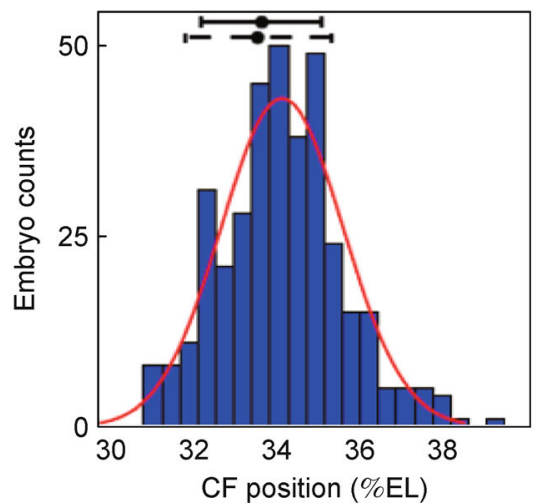

In this review, we focus on the noise transmission during the dynamic AP patterning of the fly embryos regulated by the segmentation gene network. Many other reviews provide detailed information on the other related aspects of the system [8-13]. The review is organized as the following: we first demonstrate that developmental patterning is highly dynamic. Then we discuss how this dynamics system transmits gene expression noise, and achieves the robustness against extrinsic noise such as variation of embryo length, temperature and genes. In order to reveal the underlying mechanism of noise transmission, we review the challenges in quantitative measurements and modeling on the gene network regulating AP patterning. Finally, we give a perspective of the future directions.

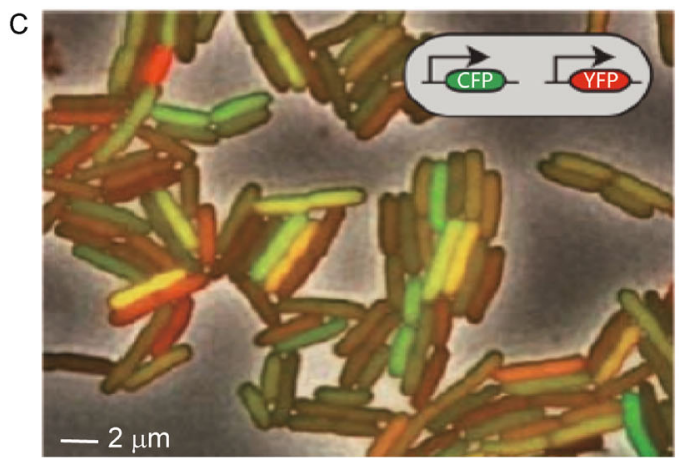

D

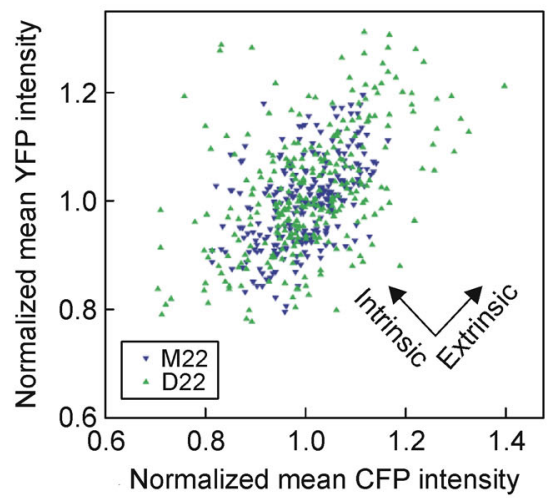

Figure 1. The contradiction between the precision and accuracy of the developmental pattern and gene expression noise at the single cell level. (A) Images of the cephalic furrow (CF) formed in the middle-coronal plane (dorsal view) of 48 fruit fly (Drosophila) embryos at $185 \pm 2$ min after fertilization at room temperature (scalar bar, $100 \mu \mathrm{m}$ ). Images were acquired on fruit flies expressing fusion protein Bcd-GFP using two-photon fluorescence microscopy. The anterior poles of all the embryos in each column are aligned on the left. The CF positions, i.e., the two intrusion bands, of the embryos in one column all align in one line (yellow line), i.e., reaching the single cell precision. (B) The histogram shows the distribution of the CF location among 364 embryos from the same fly line showing in (A). The CF position is $34.3 \pm 1.2 \%$ (mean \pm standard deviation) from a Gaussian fitting (red line). It is consistent with the CF position of the wild-type embryos (33.7 $\pm 1.7 \%$, black error bar) and Bcd-GFP embryos $(33.8 \pm 1.4 \%$, dashed black error bar) (adapted with permission from Ref. [5]). (C) An image of bacterial (E. coli) cells expressing CFP (shown in green) and YFP (shown in red) regulated by the same promoter (scalar bar, $2 \mu \mathrm{m}$ ). The color varied among different bacterial cells, indicating there exists gene expression noise (reprinted with permission from Ref. [6]). (D) The scatter plot of the normalized mean YFP intensity versus the normalized mean CFP intensity shows intrinsic noise and extrinsic noise of gene expression in two $E$. coli strains (M22 and D22) (reprinted with permission from Ref. [7]). 


\section{DYNAMIC PATTERN FORMATION DURING THE EARLY DEVELOPMENT OF FLY EMBRYOS}

The achievement of the high reproducibility and accuracy of the developmental patterns becomes an even more daunting task for a fly embryo, as it is believed to establish its blueprint of the whole body at the single-cell level in the first 3-h development after the embryo is deposited (AED). In such a short time, the developmental patterns are highly dynamic and the temporal averaging is rather limited, hence it is even more challenging to cope with gene expression noise at the single-cell level.

During the 3-h development, the fly embryo starts from one single nucleus in the center of the embryo to about 6,000 nuclei on the outer-layer of the embryo after 13 rapid nuclear divisions. To accelerate the proliferation, no cell membrane forms, and only the synthesis (S) phase and mitosis $(\mathrm{M})$ phase alternates without gap $(\mathrm{G})$ phases. The $\mathrm{M}$ phase is synchronized between different nuclei and lasts only about $3.4 \mathrm{~min}$, whereas the $\mathrm{S}$ phase remains 5 min from nuclear cycle 1 (nc1) to nc8, gradually elongates from 9 min to 13 min during $\mathrm{nc} 9$ to $\mathrm{nc} 13$, and reaches 50 $\min$ in nc14. At the $\mathrm{S}$ phase of nc10, 2/3 of the nuclei migrate from the yolk to the cortical layer of the embryo [14].

Along with the rapid nuclear division and migration process, the cell fate is determined under the regulation of the gene network. The segmentation gene network regulating the AP patterning during early fly embryogenesis has a hierarchical structure. It consists of maternal genes, gap genes, pair rule genes and segment-polarity genes from upstream to downstream. The upstream genes can regulate the expression of downstream genes, and the genes in the same class can cross-regulate with each other, but the downstream genes have no feedback to upstream genes [12]. As the gene expression feeds forward, more and more refined developmental pattern forms. At the beginning, maternal factors such as Bicoid (Bcd) $[15,16]$ and Nanos (Nos) [17] form gradients along the AP axis across the whole embryo (Figure 2B left). Subsequently, the expression domains of gap proteins such as Krupple $(\mathrm{Kr})$ and Knirps (Kni) usually span the width of 10-20 nuclei (Figure 2B middle) $[12,18]$. Each pair rule gene forms 7 three-nuclear wide stripes (Figure 2B right) [19]. Finally, each segment-polarity gene forms 14 stripes with a single-cell width, which determines the boundaries of the body segments [12].

These gene expression patterns are highly dynamic. Figure 2C illustrates the protein profiles of $\mathrm{Bcd}$ [20], $\mathrm{Hb}$ [18] and Eve [19] at different stages. The dynamics arises from a delicate spatial-temporal control of the production, degradation, and diffusion of the segmentation proteins. As a maternal factor, $b c d$ mRNAs are deposited at the anterior pole of the embryo during egg formation (oogenesis) and are locally translated with a production rate $k_{0} \mathrm{AED}$. The newly synthesized Bcd proteins diffuse away from the source with a diffusion constant $D$, and are uniformly degraded with the protein lifetime $\tau$. This formation process of Bcd gradients is usually described with the synthesis-diffusion-degradation (SDD) model, which has been discussed extensively in recent reviews $[8,21]$. According to this model, the steady state of the concentration gradient of $\operatorname{Bcd}$ is $C(x)=C_{0} * e^{-x / \lambda}$, where the amplitude $C_{0}=k_{0} / \sqrt{D / \tau}$ and the length constant $\lambda=\sqrt{D \tau}$. The Bcd gradient at location $x$ approaches its steady state with a local relaxation time $\tau_{x}=\frac{\tau}{2}\left(1+\frac{x}{\lambda}\right)$ [22]. However, it is still under debate whether the Bcd gradient agrees with the SDD model [21]. One of the big reasons is the inconsistence in the measurement of $\tau$ and $D$. Utilizing Dronpa, a photon-activated fluorescence protein, to tag with $\mathrm{Bcd}, \tau$ was measured to be $\sim 50 \mathrm{~min}$ and $\sim 15$ min before and after nc14, respectively [23]. On the other hand, with quantitative immunoblotting of Bcd from embryonic extraction, $\tau$ was estimated to be $\sim 25$ min and 55 min before and after nc14, respectively [24]. $D$ was measured to be $0.3 \mu \mathrm{m}^{2} / \mathrm{s}$ with fluorescence recovering after photon bleaching (FRAP) [2], or $7 \mu \mathrm{m}^{2} / \mathrm{s}$ with fluorescence correlation spectroscopy (FCS) [25]. Moreover, with quantitative Western blots, the total Bcd protein is found to peak at around nc12-14 [23]. Fluorescence imaging shows that the nuclear Bcd gradient starts to accumulate from $45 \mathrm{~min}$ AED and reaches the peak intensity at about nc12 ( 100 min AED) [20]. Although the nuclear Bcd intensity is the most relevant to the transcription activity of Bcd, the steady state of the nuclear Bcd gradient is further complicated given that the nuclear number and size keeps changing in different cycles and the Bcd proteins are imported into the nuclei at the beginning of each cycle and released during the mitosis [2]. Furthermore, both real-time polymerase chain reaction [23] and fluorescence in situ hybridization (FISH) [20] experiments reveal that the $b c d$ mRNA starts degradation at early nc14, and it is also suggested that the translation efficiency of $b c d$ mRNAs increases due to the modification on the $3^{\prime}$ poly-A tail before nc14 [20], hence the production rate of $\mathrm{Bcd}$ proteins also varies as development progresses $[20,23,24]$. All these factors complicate the dynamic formation of Bcd gradients, making it difficult to judge whether the Bcd gradient reaches a steady state.

Besides the dynamic inputs, the rewiring of the regulation network at different development stages also contributes to the highly dynamic expression profile of the zygotic segmentation genes. Before the middle of nc13, most of the newly synthesized proteins are maternal factors and they are the main inputs to regulate the 
A
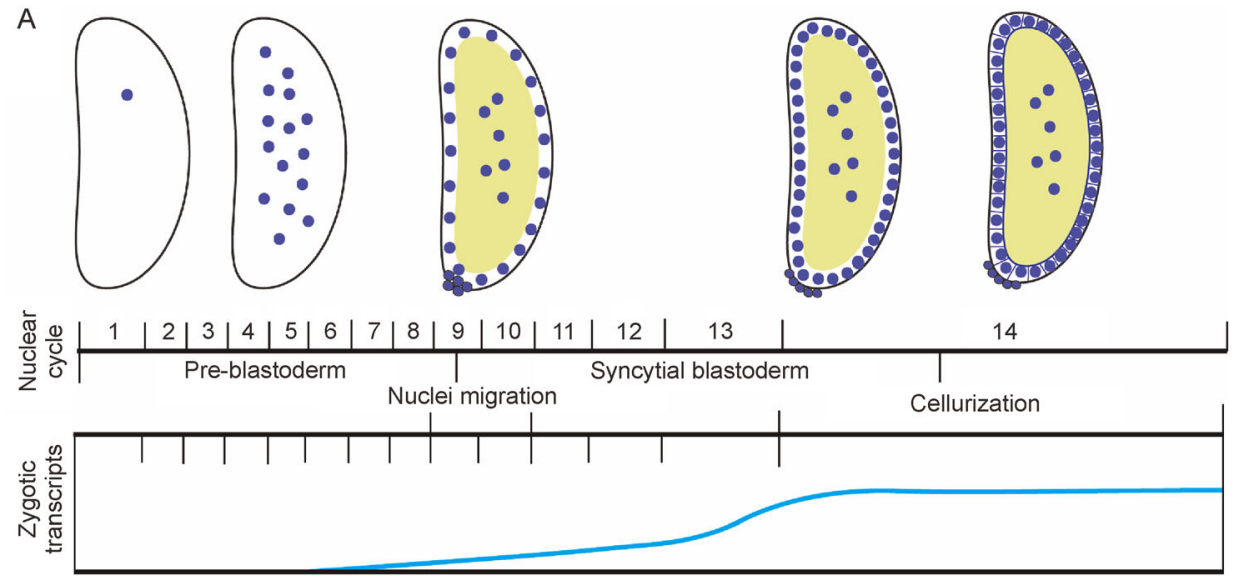

B
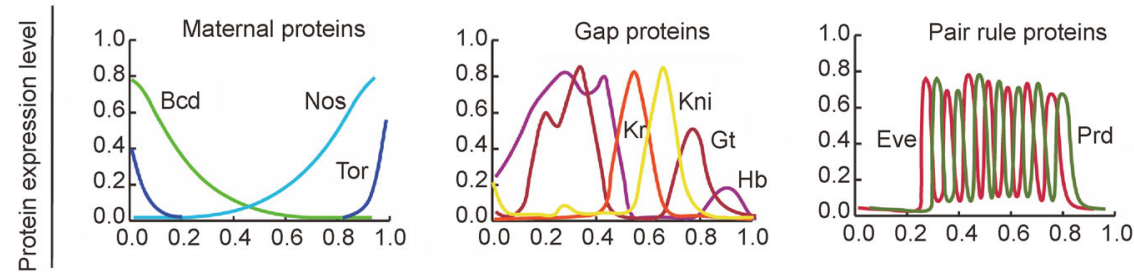

C

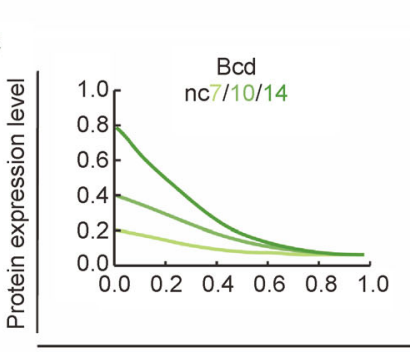

Embryo length (x/L)
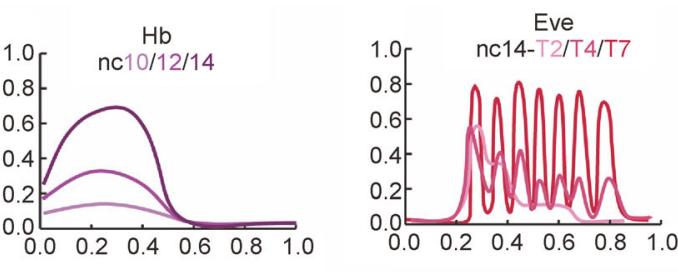

Embryo length (x/L)

D

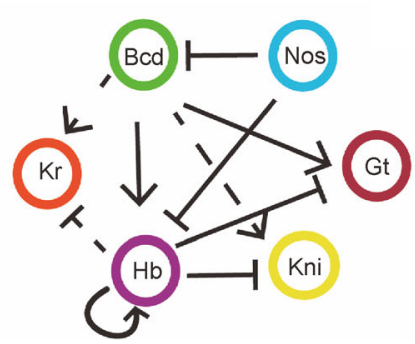

\section{E}

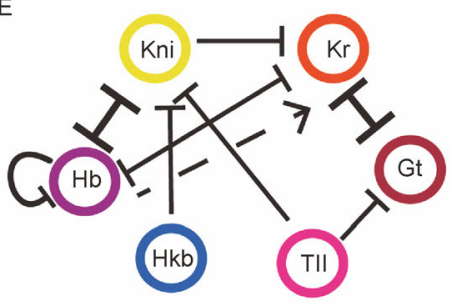

Figure 2. Dynamics of development patterns during early Drosophila embryogenesis (A) The dynamics of the nuclear position, nuclear cycle and zygotic transcription activity of fly embryos before nuclear cycle 14 (nc14). After fertilization, the nuclei divide for nine times in the yolk of the embryo. Then two-thirds of the nuclei migrate to the surface of the embryo and divide for another four times. At nc 9 , pole cells start to form in the posterior pole. The nuclear cycle only has the $S$ and $M$ phase before $\mathrm{nc} 14$. The duration of the $\mathrm{M}$ phase remains at $3.4 \mathrm{~min}$, whereas the $\mathrm{S}$ phase elongates from $5 \mathrm{~min}$ in $\mathrm{nc} 1-8$ to $9 \mathrm{~min}$ and $13 \mathrm{~min}$ in $\mathrm{nc} 9$ and nc13, respectively. It is further lengthened to be 50 min in nc14. The zygotic gene expression level is low before nc13 and increases significantly during maternal zygotic transition (MZT) in nc13-14. (B) The segmentation gene network regulating the anterior-posterior (AP) patterning during early Drosophila embryogenesis starts from the formation of the protein gradients of the maternal factors including Bcd (green), Nos (light blue), and Tor (dark blue) (left), to the expression of gap genes including $h b$ (purple), gt (scarlet), kni (yellow) and $K r$ (orange) (middle), pair rule genes including eve (red) and pr (light cyan) and finally the segment-polarity genes (not shown). The sketch of these gene expression patterns is drawn to reflect the spatial distribution of the normalized concentrations at their highest expression level. (C) The dynamics of the protein profiles of Bcd (left), $\mathrm{Hb}$ (middle) and Eve (right). (T\# represents the \# time period equally dividing $n c 14$ for 8 times). ( $D-E)$ The AP patterning segmentation gene network switches from the maternal driven before maternal zygotic transition (MZT) (D) to the strong cross-regulation between gap genes after MZT (E) during early fly embryogenesis. The width of the line indicates regulation strength. Dashed lines indicate the regulation relationship remains to be confirmed. 
downstream zygotic gene expression (Figure 2D) [12]. After nc13, the zygotic gene expression level increases significantly (Figure 2A) [14] and the cross regulation between the zygotic genes becomes dominate, meantime the maternal inputs shut down timely (Figure 2E) [12,26]. This process is called maternal zygotic transition (MZT). For instance, $\mathrm{Hb}$ has both maternal and zygotic components. At early developmental stages, the translation of the evenly distributed maternal $h b$ mRNA is repressed by the Nos gradient [17], resulting a stepfunction like $\mathrm{Hb}$ profile with a gradual boundary in the middle of the embryo. During nc12-13 and early nc14, as $\mathrm{Bcd}$ and $\mathrm{Hb}$ activate the zygotic expression of $h b$, the $\mathrm{Hb}$ boundary sharpens and the expression level at the anterior half increases [18]. Later, while the zygotic expression of $h b$ becomes much higher than the maternal $\mathrm{Hb}$, the enhancer regulating the zygotic expression gradually switches from Bcd-dependence to Bcd-independence $[26,27]$, the self-regulation by $\mathrm{Hb}$ itself changes from activation [28-30] to repression [31], and the repression by the other gap genes $k n i$ and $K r$ becomes more significant $[31,32]$, consequently the transcription profile changes from a step function to two stripes [27,33]. One forms at the boundary in the middle of the embryo, resulting in further boundary sharpening [31], accordingly the concentration of the anterior profile arises to the highest level at nc14-T7 ( 42 min AED) then gradually drops down [18]. The other one is the rising posterior stripe of $\mathrm{Hb}[27,34]$. As a result, the $h b$ expression profile constantly evolves and shows no steady state (Figure 2C middle).

As the downstream genes in the hierarchical segmentation gene network, the pair rule genes and segmentpolarity genes also show dynamic evolved expression profiles. For instance, starting from a broad single peak in nc13, eve transiently reaches the highest expression level and shows 7 strips with even spacing and equal height at nc14-T7 (Figure 2C right), then gradually disappears [19].

\section{NOISE TRANSMISSION DURING THE DYNAMIC DEVELOPMENT PROCESS}

It is generally believed that the cascades of the dynamical gene expression of segmentation genes lead the cell fate determination at the single-cell level, i.e., each cell has its unique gene expression when the fly embryo starts gastrulation at about $4 \mathrm{~h}$ AED. In other words, the gene expression profiles provide sufficiently precise positional information for each cell to "know" its position in the embryo, so it can differentiate and migrate to form the tissue and organ accordingly [35]. However, gene expression noise usually deteriorates the positional information carried by the gene expression profile. It is intriguing how the fly embryo overcomes gene expression noise to achieve single-cell precision in developmental patterning. Two scenarios have been proposed regarding noise transmission during the dynamic developmental process [36]. Hypothesis I suggests a "channel"-like transmission, i.e., the system starts with a highly precise input, and accurately transmits the positional information from upper class to lower class (Figure 3A) [36]. This scenario is based on the "French flag model", i.e., the cell fate is determined by a global morphogen concentration gradient, which activates a series of genes at different thresholds [10,37]. It assumes an accurate and precise morphogen gradient is established and subsequently accurately interpreted by the downstream genes $[1,36,38]$. Hypothesis II suggests a "filter"-like transmission, i.e., the system starts with a noisy input but the positional noise gradually decreases via a "filter" mechanism during its transmission [39]. Such a "filter" mechanism can emerge through the cross-regulation between the genes of a "self-organized" network [40,41]. A famous example is Turing pattern formation. A nonlinear dynamic process (autocatalysis and reactant inhibition) coupling with a special diffusion process (a slow diffusing activator and a fast diffusing inhibitor) can generate periodic oscillation patterns (Figure 3A) [42,43]. It has been under great debates in previous studies which scenario the fly embryo follows. One of the main reasons is the controversy in measuring the noise of $\mathrm{Bcd}$ gradients.

Some studies seem to support the "filter" hypothesis. Houchmandzadeh et al. compared the positional error of the morphogen $\mathrm{Bcd}$ and its downstream $\mathrm{Hb}$ in nc14 using the immunofluorescent staining method [39]. The boundary position of the anterior profile of $\mathrm{Hb}, x_{H b}$, was defined as the location where the normalized intensity is 0.5 . The measured standard deviation of $x_{H b}$ from about 100 wild type (WT) embryos was $1 \%$ L. In contrast, the position of the normalized Bcd profile at a fixed threshold of 0.23 , which is corresponding to $x_{H b}$, showed a variability range of 30\% L and a standard deviation of 7\% L (Figure 3C). These results suggest that the noise of Bcd gradients must be filtered out to achieve the high positional precision of $\mathrm{Hb}$ boundary. Subsequently, the cross-regulation between gap genes were reported to "canalize" the patterning, improving the precision and driving the dynamical shifts of patterns $[40,41]$.

However, other studies challenge the "filter" hypothesis and favor the "channel" hypothesis. With live imaging of the Bcd gradient and carefully designed control experiments, Gregor et al. demonstrated that the Bcd gradient was highly reproducible [1]. In the anterior region, the gradient noise, i.e., the relative standard deviation of the Bcd expression level at the same position in different embryos, is $\sim 10 \%$, which corresponds to a positional error 

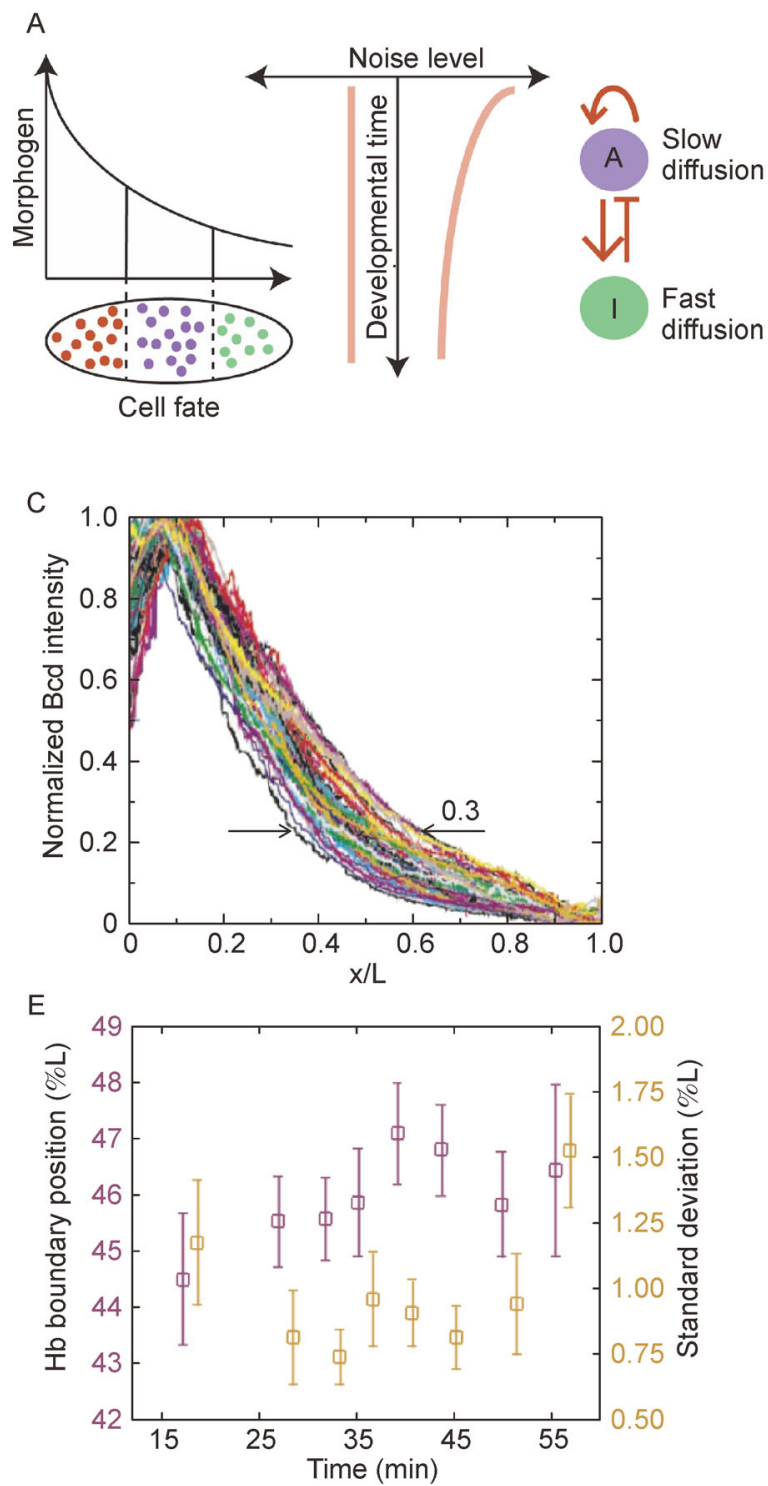

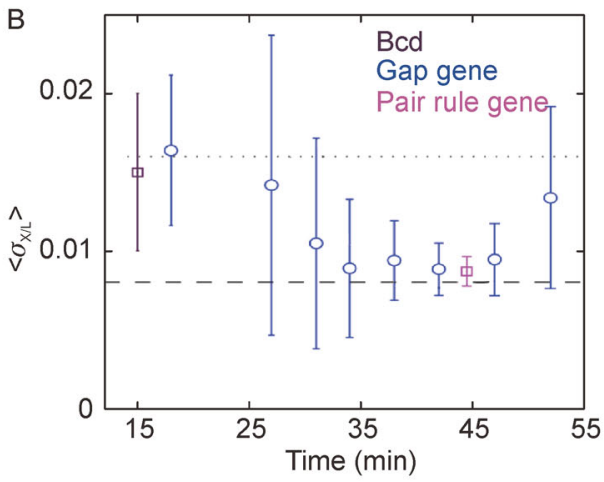

D

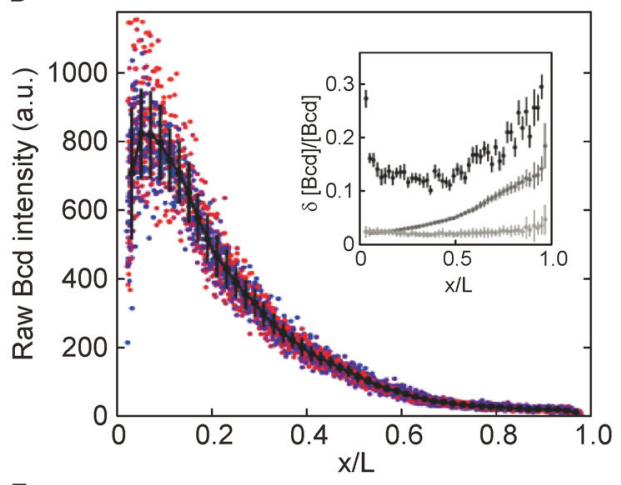

$\mathrm{F}$

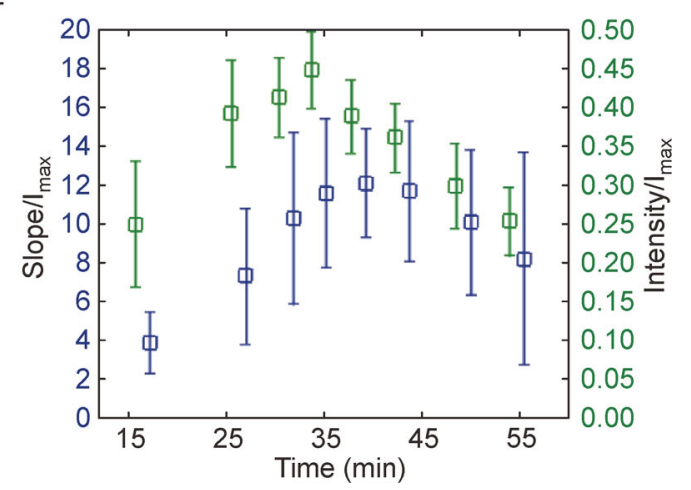

Figure 3. Noise transmission during the dynamic developmental processes. (A) Two noise transmission hypotheses. On the left, the cell fate pattern is determined by the French flag model, i,e., a morphogen gradient, e.g., Bcd, activates different genes at different thresholds. Ideally, the noise level shows a channel-like transmission curve if the downstream gene can accurately read the position information from the upstream genes. On the right, the gene network has a certain noise filtration mechanism similar to self-organization process in Turing pattern formation. (B) Temporal evolution of the reproducibility of the segment gene expression profiles during nc14. The positional error of the markers of the gap genes (blue circle) gradually decreases to reaches the minimum at around 40 min into nc14. It is consistent with the minimum of the positional error of its upstream Bcd gradient (violent square) at 15 min into nc14 (adapted with permission from Ref. [18]). It also matches with the positional error of its downstream pair-rule gene (pink square) at 45 min into nc14 Ref. [18]. The error of the CF position is comparable with the minimum value of the gap genes [5]. (C) The normalized immunostained Bcd expression profile from different embryos (represented by different colors) show huge variation corresponding to $30 \%$ positional error in the middle of the embryo (adapted with permission from Ref. [39]). (D) The raw fluorescence intensity of the Bcd-GFP gradient along the AP axis using two-photon fluorescence microscopy shows much less gradient noise in the anterior half of the embryo. Each color represents different embryos. Each dot represents the average fluorescence intensity of the nucleus. Error bars represent the mean and standard deviation of fluorescence intensity in each bin with the bin width of $2 \%$ embryo length (L). Inset shows the relative standard deviation (standard deviation/mean) as a function of embryo length for each bin (upper) and the imaging noise and image processing noise (lower) (adapted with permission from Ref. [5]). (E) The mean (violet square) and the standard deviation (yellow square) of the position of the $\mathrm{Hb}$ boundary as a function of time in nc14. 89 embryos are divided into 8 equal-samplesized bins according to their ages. Error bars of the mean position show standard deviation in each bin. Error bars of the standard deviation are calculated via bootstrap sampling. (F) The average slope (blue square) of the $\mathrm{Hb}$ boundary and the corresponding intensity at the $\mathrm{Hb}$ boundary positions (green square) as a function of time in nc14. Raw $\mathrm{Hb}$ intensity is normalized by the maximum intensity among all the embryos. Error bars represent standard deviation of the slope or intensity in each bin. 
of $1 \%-2 \% \mathrm{~L}$ based on the error propagation calculation. Furthermore, by improving the signal to noise ratio of imaging, the gradient noise of Bcd was actually 10\%$20 \%$ in almost the whole embryo (Figure 3F) [5]. And similar results were observed in the measurement using immunofluorescence staining [38]. The discrepancy between these results and previous results arises from the different measurement errors (for more detailed analysis see the section after next). In particular, the greater measurement errors in Houchmandzadeh's measurement are mainly attributed to the improper normalization as the gradient noise was shown to approach the level of $10 \% \mathrm{~L}$ after a better renormalization [11]. Hence the positional error derived from the gradient noise of $\mathrm{Bcd}$ based on error propagation is about $1 \%-2 \% \mathrm{~L}$, which is almost the same level as that of the $\mathrm{Hb}$ boundary. Furthermore, the input/output relation between Bcd and $\mathrm{Hb}$ was measured in the same embryo with immunofluorescent staining. It could be fitted with the Hill function where the Hill coefficient $n=5$. Assuming the $\mathrm{Hb}$ level is a direct readout of the Bcd gradient, the input noise in Bcd concentrations can be converted from the measured output noise in $\mathrm{Hb}$ levels based on the measured input/output function of $\mathrm{Bcd} / \mathrm{Hb}$. And the gradient noise of Bcd approaches its minimum value of $\sim 10 \%$ at the $\mathrm{Hb}$ boundary $x_{H b}$. Hence the $\mathrm{Hb}$ noise was suggested to directly transmitted from the Bcd gradient without any "filtering" mechanism [1].

However, it is still debatable that the Bcd gradient alone is able to convey sufficient positional information to $\mathrm{Hb}$. Both Bcd gradients and $\mathrm{Hb}$ profiles are highly dynamic. In fact, as the overall intensity, the average position and slope of the boundary of the $\mathrm{Hb}$ profile reach the maximum values, the standard deviation of the $\mathrm{Hb}$ boundary reaches the minimum value at around $40 \mathrm{~min}$ into nc14 (Figure 3E-F) [18]. If one calculated the standard deviation of all the gap gene markers such as the signature boundaries or peak positions, it actually follows a similar trend: the positional error gradually decreases from about $2 \% \mathrm{~L}$ at the beginning of nc14 to the minimum value of $0.75 \% \mathrm{~L}$ at $\sim 45 \mathrm{~min}$ into nc14. Interestingly, the combined positional error of gap genes at the beginning of nc14 is comparable with the one converted from the gradient noise of Bcd measured at about 15 min into nc14 (Figure 3D) $[1,5,38]$. And the minimum positional error of gap genes is consistent with the one of the pair rule genes (Figure 3B) [18].

Taking together, the positional information is carefully controlled at each level of the hierarchical segmentation gene network. Starting with the highly reproducible Bcd gradient, the positional information is slightly refined by a factor of 2 to the single-cell precision in gap gene profiles, subsequently is relayed by the pair rule genes and etc.
Thus the fly embryo development might strictly fit into neither the originally proposed "filter" nor "channel" scenario. More rigorous experiments are still in quest to test whether it has the capability to utilize both mechanisms to achieve the positional precision at the single-cell level.

\section{PATTERNING ROBUSTNESS AGAINST THE EXTRINSIC NOISE: VARIATION OF THE EMBRYO LENGTH, TEMPERATURE AND GENES}

The dynamic development patterning not only copes with the gene expression noise, but also achieves the robustness in response to the extrinsic noise such as environmental fluctuations and genetic variation.

AP patterning of fly embryos is spatially scaled with embryo length $[39,44]$. The natural embryo length variation is $\sim 4 \%$ [1]. The variability of embryo lengths in a population can increase further through temperature changes. For example, the embryo length of Ore-R flies decreases from $524 \pm 25 \mu \mathrm{m}$ at $18^{\circ} \mathrm{C}$ to $480 \pm 14 \mu \mathrm{m}$ at $29^{\circ} \mathrm{C}$. Hence, to achieve AP patterning with $1 \% \mathrm{~L}$ precision, scaling is a necessary property to maintain the robustness against the variation of embryo length. Indeed, the signature boundaries or peaks of AP patterning, e.g., the posterior boundary of the anterior $\mathrm{Hb}$ profile, are positively proportional to the embryo length (Figure 4A) [39]. However, it is still very controversial whether the Bcd gradient is scaled with the embryo length. Based on the SDD model, the diffusion-driven Bcd gradient is hypothetically unscaled with embryo length. This leaves the origin of spatial scaling of developmental patterns an enigmatic question. Several hypotheses have been proposed to solve this mystery. For instance, the nuclear trapping model [2] predicts that Bcd gradients have a scaled length constant, but several lines of experimental evidence contradict such a prediction [45]. It has also been hypothesized that scaled Bcd gradients are generated by volume-dependent modulation of the amplitude of the Bcd gradient [46], which is originated from oogenesis as proposed in the $\mathrm{TEM}^{3} \mathrm{~S}$ (Tissue Expansion-Modulated Maternal Morphogen Scaling) model [47], but further experimental evidence needs to show that this is sufficient to generate the scaling of downstream genes. One study reported that the positional precision of Bcd-target genes mirrors that of Bcd [48], suggesting that the precision and scaling of Bcd-targeted genes were generated by presteady-state decoding of Bcd gradients [49]. However, it has been questioned whether such decoding exists in the AP patterning system [50]. The bi-gradient model assumes a posterior gradient interacts with the $\mathrm{Bcd}$ 


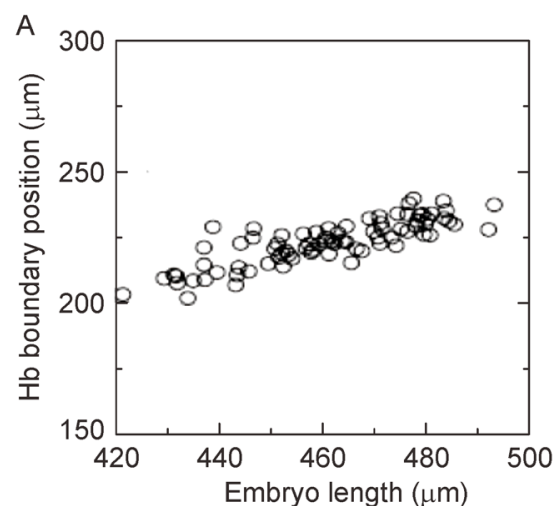

D

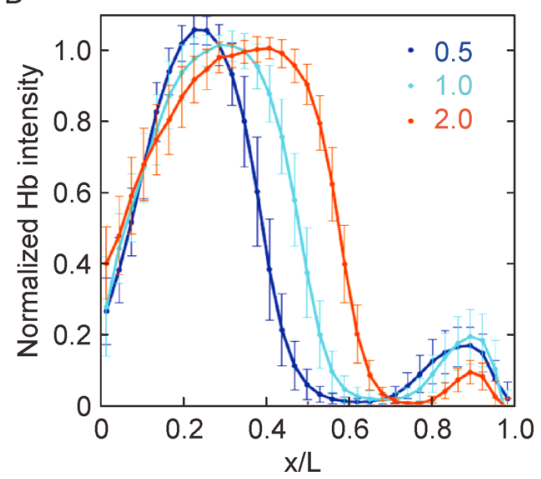

B

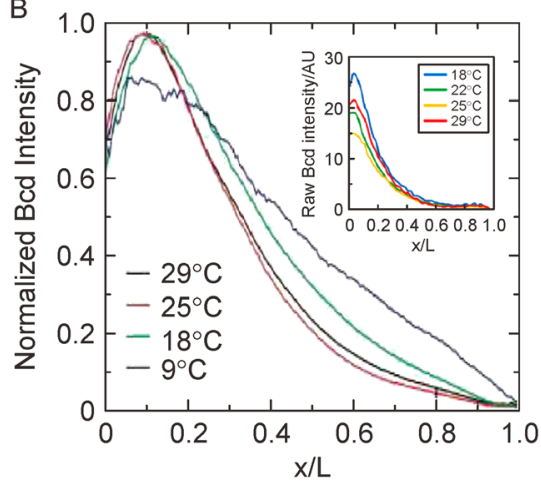

$\mathrm{E}$

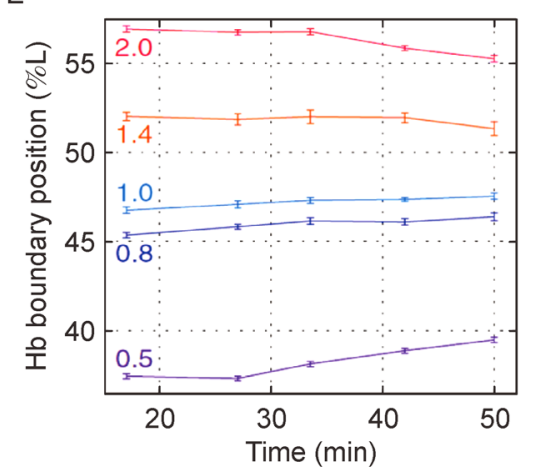

C

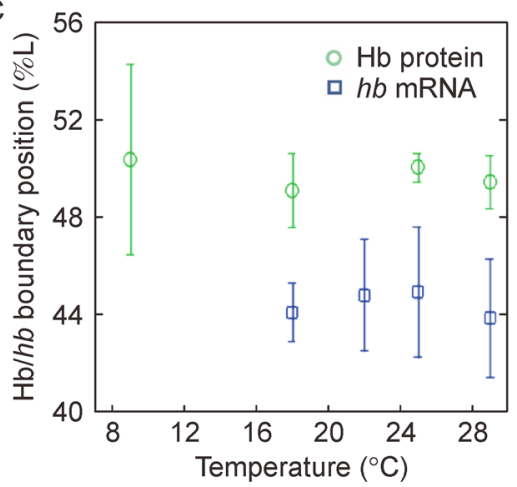

$\mathrm{F}$

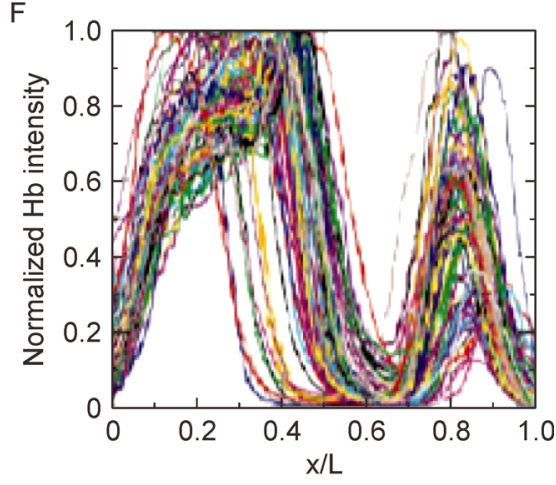

Figure 4. The robustness of $\mathrm{Hb}$ boundary positions. (A) The absolute position of the $\mathrm{Hb}$ boundary measured from the anterior pole is scaled with the embryo length. Data was collected from fixed embryos of the WT fly line (adapted with permission from Ref. [39]). (B) As temperature varies, the shape [39] and the amplitude (inset, adapted with permission from Ref. [53]) of Bcd gradients changes. (C) As temperature varies, the mean positions of the boundaries of $\mathrm{Hb}$ protein (green circle) and $h b$ mRNA (blue square) profiles remain constantly. Error bars represent the standard deviations of the mean. (D) Average dorsal Hb profiles of embryos fixed at 10-24 mins into nc14 from fly lines with Bcd dosages of $D=0.5$ (blue), 1.0 (cyan), and 2.0 (orange). For each line, profiles were binned into 33 equispaced bins along the AP axis. Lines are the mean profile intensity in each bin. Error bars represent the standard deviation of the profile intensity in each bin (reprinted with permission from Ref. [5]) (E) The mean position of the Hb boundary, $x_{H b}$, as a function of time in nc14 for embryos with Bcd dosages of $D=0.5$ (dark blue), 0.8 (light blue), 1.0 (cyan), 1.4 (light orange), and 2.0 (orange). Error bars represent the standard error of the mean. Time is measured from the beginning of nc14, with each value on the time axis representing the average time in each time class (reprinted with permission from Ref. [5]). (F) The normalized $\mathrm{Hb}$ protein profiles detected by immunofluorescence staining in nc14 from stau ${ }^{H L}$ embryos (reprinted with permission from Ref. [39]).

gradient to generate scaled center developmental patterns [51,52], however, the molecular basis for the posterior gradient is still unclear. Future quantitative measurements are still in quest to understand the underlying mechanism for the spatial scaling of segmentation patterns during early embryogenesis, in particular, whether the Bcd gradient is able to precisely provide scaling information for embryos of varying sizes.

AP patterning is robust in response to temperature fluctuations. The temperature has profound effect on a reaction-diffusion system such as embryonic patterning, since the diffusion, degradation, and interactions of the biological molecules in the embryos are all temperature dependent. In fact, the developmental rate increases as the temperature augments [54]. For example, the hatching time decreases from 18 days in $19^{\circ} \mathrm{C}$ to 7 days in $29^{\circ} \mathrm{C}$. In addition, the embryo length increases at lower temperatures. However, the average position of boundaries of the $\mathrm{Hb}$ protein and $h b$ mRNA profiles are observed to be constant for embryos developed at different temperatures in the range of $9^{\circ} \mathrm{C}$ to $29^{\circ} \mathrm{C}$ (Figure 4C) [39]. Even more surprisingly, fly embryos can counteract the effects of a time-dependent temperature step, i.e., the anterior and the posterior halves develop in different temperatures, in a microfluidic device [55]. Both $\mathrm{Hb}$ and Eve profiles were normal even if the temperature step was switched in a period except that the switching period was between 65 $100 \mathrm{~min}$ AED [55]. These results suggest that a temperature compensation mechanism accounts for the robustness against the temperature fluctuations and it might act in a critical developmental window. But the molecular basis for such a temperature compensation 
mechanism is still under hot debate. Several studies show that the average Bcd gradients show greater [39] or slightly smaller [53] length constants, and greater amplitudes [53] at temperatures other than $25^{\circ} \mathrm{C}$ (Figure $4 \mathrm{~B}$ ), and the Bcd gradient is severely distorted during nc11-13 in the microfluidic device [56]. Hence the precise input from the Bcd gradient was suggested to be nonessential for the temperature-independent precise AP patterning such as $\mathrm{Hb}$ profiles. One study proposed that a bi-gradient consists of the Bcd gradient and the other unknown posterior gradient could generate invariable $\mathrm{Hb}$ boundaries in the middle of the embryo [51]. The compensation mechanism was also suggested to be attributed to some endo-siRNAs which might regulate the temperature-induced change of the nuclear cycle based on the measurement of mutant flies in the temperature-step experiment [57]. On the other hand, a more recent study argued that the Bcd concentration threshold at the $\mathrm{Hb}$ boundary was relatively robust as the temperature varied in a certain range [53]. To clarify the confusion, more quantitative measurements on the temperature effect on the precision of the AP patterning are still needed in future studies.

AP patterning is also robust in response to genetic variation. One excellent example is the $\mathrm{Hb}$ boundary. Nearly each gene related with $\mathrm{Hb}$ regulation was mutated such as knocking out nos and $K r$ or removing selfactivation of $h b$, even one by one a half arm of the chromosome comprising nearly $5 / 6$ of the whole genome was removed, the mean or variability of the $\mathrm{Hb}$ boundary position show no significant change [39]. The $\mathrm{Hb}$ boundary does shift if we manipulate the Bcd dosages (Figure 4D). However, during nc14 the shift amount dynamically decreases from $\sim 100 \%$ to $\sim 60 \%$ of the predicted shift based on the threshold-dependent model assuming Bcd is the only input (Figure 4E). Note the variability of the $\mathrm{Hb}$ boundary remains nearly unchanged upon the alteration of the Bcd dosage [5]. So far only staufen (stau) knockout has been discovered to dramatically increase the variability of the boundary of $h b$ mRNAs [58] and Hb proteins [39] (Figure 4F). Strangely, Stau has not been discovered to directly regulate gene expression. It is best known as a localization factor for bcd mRNAs and nos mRNAs [59]. Several models were proposed to explain the function of Stau in patterning $\mathrm{Hb}$ boundaries without any supporting experimental evidence [60]. One experiment showed that the gradient noise of Bcd increases in the stau mutant fly line and suggested this could account for the increased variability of the $\mathrm{Hb}$ boundary [38].

To summarize, the precision of the $\mathrm{Hb}$ boundary is observed to be robust under the perturbation of the embryo length, temperature or genes. But it is still unclear whether this robustness against the extrinsic noise stems from the adaptive upstream maternal morphogen gradients according to the "channel" hypothesis or the interaction between the downstream segmentation genes according to the "filter" hypothesis.

\section{CHALLENGES IN QUANTITATIVE MEASUREMENTS AND MODELING ON DYNAMIC DEVELOPMENTAL PATTERNS}

To test which hypothesis agrees on the noise transmission during the dynamic development of the fly embryo, it is crucial to carry out quantitative measurements on AP patterning. However, it has been very challenging to quantify the noise of the gene expression profiles during early embryogenesis. Since the gene expression patterns constantly evolve dynamically (Figure 2C) in a 3D asymmetrical embryo, accurate extraction of the average and noise of the gene expression at the single-nucleus level requires carefully identifying and controlling the temporal and spatial measurement errors. Two types of quantitative imaging methods have been developed. One is live imaging proteins tagged with fluorescence proteins [2,5] or mRNAs with the MS2-MCP system [61,62] on living embryos from transgenic fly lines; the other is imaging on fixed embryos with specifically marked proteins with the immunofluorescence staining technique [19] or mRNAs with the FISH techniques [20,63,64].

From the dynamic point of view, live imaging seems to have a great advantage in controlling the temporal measurement errors. We can choose a desired time point for measurements by clocking down from a reference time point, e.g., the beginning or end of a mitosis between two nuclear cycles (Figure 2A), with at least 1-min time resolution. However, one needs to check whether the observed fluorescence dynamics faithfully recapitulates the actual biological dynamics $[2,65]$, as it is well known that genetic modification (e.g., random insertion) in generating the transgenic fly lines or phototoxicity during imaging might disturb the physiology of the biological system, and the diffusion and/or degradation of the proteins tagged with fluorescence proteins may differ from that of the endogenous species [66-68]. Furthermore, the maturation of the fluorescent protein often causes the distortion of the observed protein expression profile. For instance, the Bcd-GFP gradient was measured in a living embryo, subsequently in the same embryo after it was immediately fixed and hand-peeled (which reserves the fluorophore of eGFP) with the same imaging condition. Besides about 3 times increase of the overall fluorescence intensity, the intensity discrepancy of the Bcd-GFP gradient before and after fixation is greater as the position is closer to the anterior pole (Figure 5A) [20]. This distortion can be well explained if we add the maturation of eGFP into the SDD model [5] (Figure 5B). 
Since it takes tens of minutes for eGFP to mature inside the embryo, only a small fraction of the newly translated Bcd-GFP proteins show fluorescence close to their source, the anterior pole, and as they diffuse away more and more convert from the dark state to the fluorescent state [20] (Figure 5B). Thus in live imaging, the apparent length constant of the observed Bcd-GFP gradient is greater than the real one, and the measured noise could be more than the true biological noise because of the stochastic maturation process. Based on the modified SDD model, the calculated correction factors for BcdGFP gradients are as great as 3 at the anterior pole by comparing the measured Bcd-GFP gradients from the imaging with the living embryos and fixed embryos (Figure 5B inset). However, a universal method to correct the maturation effect for other genes is still in demand. Theoretically, one could build a mathematical correction model given the maturation time of the fluorophore and the degradation time of the protein. However, the two parameters vary in different living systems and are difficult to measure directly. Moreover, the fluorescence signal could be even below the detection limit of live imaging if the protein degradation rate is much greater than the maturation rate.

To control the temporal measurement errors for imaging on fixed embryos, several methods have been developed to estimate the developmental time based on the signature of the gene expression profile or embryo morphology. For example, eve has been often chosen as the reference gene for embryo ages [40,69], and their expression profiles in nc14 can be classified into 8 temporal windows with the width of $6.5 \mathrm{~min}$ (Figure 2C, right). As one of the best-characterized morphological signatures, the depth of the membrane furrow canal can be utilized to determine the developmental time with about 2 min precision during the early $30 \mathrm{~min}$ of nc14 and less than $1 \mathrm{~min}$ during the next $30 \mathrm{~min}$ in nc14 before gastrulation (Figure 5C). To further improve the timing precision in early nc14, it has also been suggested that the nuclear height or nuclear shape could be utilized to stage embryos with $1 \mathrm{~min}$ precision in the first $10 \mathrm{~min}$ into nc14 (Figure 5C) [26]. Nevertheless, these methods are restricted in estimating developmental time in nc14. In early nuclear cycles, usually only the cycle number can be determined based on the nuclear density. Recently, a 3min time resolution has been achieved even in early nuclear cycles by fixing the embryo reaching the target developmental time from a reference time point determined by live imaging, e.g., the metaphase of the preceding nuclear cycle [70].

To control the spatial measurement error, it is important to mount the embryos in a specific orientation, e.g., the symmetric dorsal view or the asymmetric lateral view. From the lateral view, the embryos were often imaged on the midsagittal plane. For the segmentation genes such as $b c d, h b$ and eve, the protein expression profiles on the dorsal side are significantly different from those on the ventral side (Figure 5D-F). In fact, azimuthal orientations of embryos can cause spatial errors $2 \%-13 \%$ of the total variance [18]. In principle, 3D imaging based on precise nuclear registration may overcome this error. For example, 3D gene expression profiles of RNAs have been extracted with two-photon microscopy imaging on fixed embryos [71]. However, the 3D imaging on living embryos is still limited unless the imaging speed is improved.

Several other sources such as the uncertainty of the imaging plane selection, imaging noise, cross-talks between neighboring optical channels and imaging processing noise, also contribute to the measurement errors in quantitative imaging on embryos. Carefully designed control experiments show that they can account for $1 \%$ or less to the total variance [18]. As for immunofluorescence staining, nonspecific binding of primary and secondary antibodies is another source of errors, contributing $4 \%-10 \%$ to the total variance [18]. Antibodies with more specificity can help to reduce this error [72].

Taking the above factors into consideration, different imaging methods could be preferred for quantifying different gene expression noise in fly embryos based on our previous experimental experience. For example, twophoton imaging with living embryos is so far the best choice for the Bcd gradient measurements [1,5], as it often yields much higher signal to noise ratio compared with other imaging experiments [38,39], whereas the fixed embryos often show a higher level of auto-fluorescence background, and higher imaging noise due to the stochastic binding of antibodies in immunofluorescence staining. On the other hand, if we want to measure the gap gene or pair rule gene profiles, immunofluorescence staining with fixed embryos will still be a better choice. Because it has been difficult to prepare a transgenic fly line to show the normal dynamics of the gene expression of gap gens or pair rule genes with live imaging. This might not only due to the maturation effect, but the positional effect, i.e., the random insertion of the tagged genes does not faithful capture the complicated regulation of the endogenous gene. In fact, unlike the Bcd-GFP fly lines, no viable transgenic fly lines have been reported with the knockout of the corresponding endogenous gap or pair rule gene. This problem might be solved with the application of the CRISPR-Cas9 technique [73,74]. Ideally, it is always recommended to quantify the gene expression in fly embryos with more applicable methods to avoid the potential artifacts.

Quantitative mathematical models describing the dynamic gene expression profiles during early fly 
A

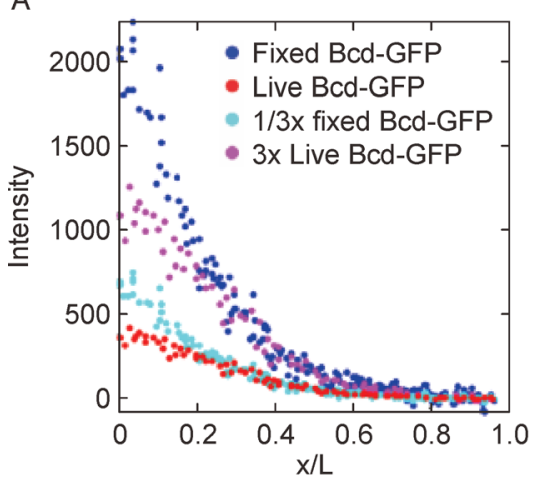

D

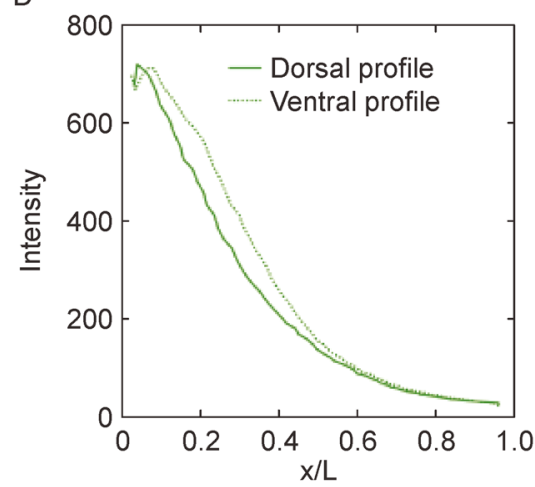

B

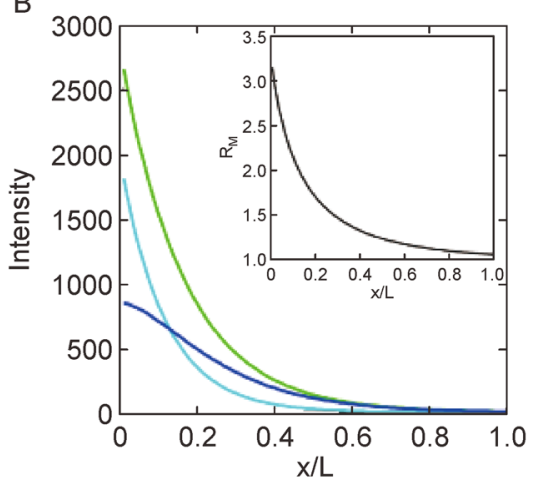

E

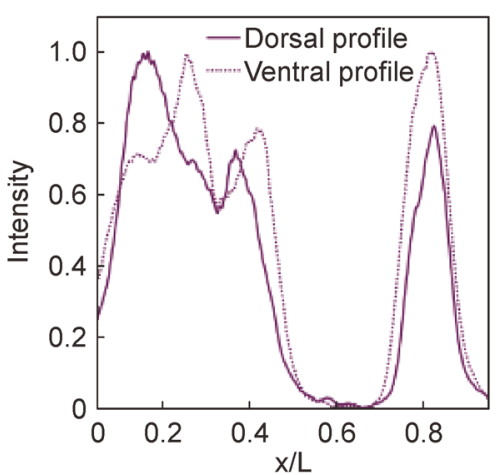

C

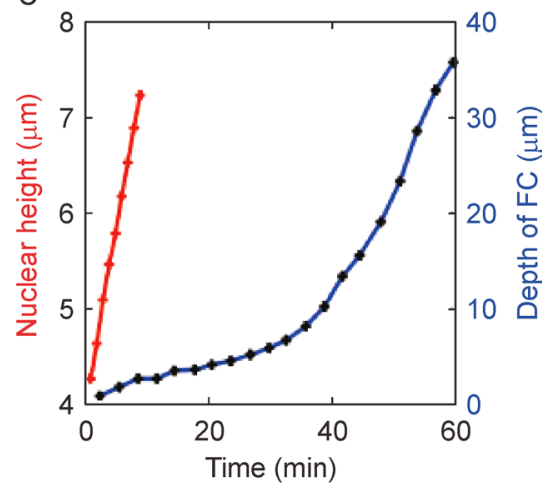

$\mathrm{F}$

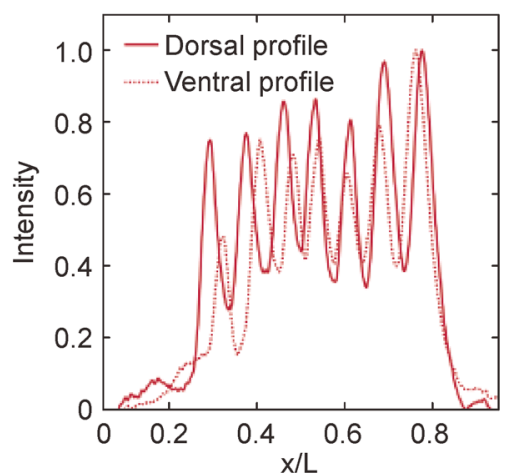

Figure 5. Origin of the temporal and spatial measurement errors in quantitative measurement of the dynamic developmental patterns in fly embryos. (A) The delayed eGFP maturation distorts the shape of the Bcd gradient in live imaging: comparison of the average nuclear fluorescence intensities in an embryo expressing Bcd-GFP in live imaging (red dots), and fixed embryos detected by immunostaining (blue dots). (B) Model correction on the eGFP maturation effect on the steady-state Bcd-GFP concentration $C$ versus $x / L$. The total concentration $C_{\text {tot }}$ (green line) is composed of $C_{m}$ (blue line, contributed by Bcd-GFP molecules with matured eGFP, which are visible with live imaging) and $C_{i m}$ (cyan line, contributed by Bcd-GFP molecules with immature eGFP, which are invisible for live imaging), assuming the degradation time of Bcd-GFP is the same as the maturation time of eGFP. Inset shows a log-linear plot of these gradients. (C) Age determination through nuclear height and depth of furrow canal (FC): nuclear height changes in the early $10 \mathrm{~min}$ into nc14 with time precision better than $1 \mathrm{~min}$ (red line), while the depth of the furrow canal changes during the whole nc14 with precision of 1-2 min (blue line). (D-F) Expression profiles vary at different embryo orientations: expression profiles of $\mathrm{Bcd}(\mathrm{D}), \mathrm{Hb}(\mathrm{E})$, Eve $(\mathrm{F})$ at the dorsal side (solid line) and the ventral side (dashed line).

embryogenesis also play an important role in unravel the underlying mechanism of noise transmission. One of the most widely applied models is the ingenious gene circuit model proposed by Reinitz group [40]. It consists of a set of ordinary differential equations modeling the protein concentration $v_{i}^{a}$ over time $t$ for the product of gap gene $a$ in nucleus $i$, governed by the protein production (with a production rate $R_{a}$ ), diffusion (with a diffusion rate $D_{a}$ ) and decay (with a decay rate $k_{a}$ ):

$$
\begin{aligned}
\frac{\mathrm{d} v_{i}^{a}}{\mathrm{~d} t}= & R_{a} g\left(\Sigma_{b=1}^{N_{g}} T^{a b} v_{i}^{b}+\Sigma_{\beta=1}^{N_{m}} E^{a \beta} v_{i}^{\beta}(t)+h^{a}\right) \\
& +D_{a}\left[v_{i-1}^{a}+v_{i+1}^{a}-2 v_{i}^{a}\right]-k_{a} v_{i}^{a} .
\end{aligned}
$$

The production is driven by the linear inputs of the interactions of gap genes (represented by matrix $T$ ), the maternal factors (represented by matrix $E$ ) and a baseline constant $h^{a}$. All of the inputs are summed up and fed into a sigmoid regulation expression function $g$. All 56 parameters are obtained by fitting to dynamic protein expression profiles of gap genes in nc14 measured with fluorescence immunofluorescence staining. It successfully captures the dynamic shifts of gap gene boundaries during the middle nc14, and reveals that this dynamics is attributed to the asymmetric cross-regulations between the gap genes. Nonlinear analysis based on this model suggests two different types of attractors account for anterior and posterior profiles of $\mathrm{Hb}$ [41].

Different from the data-driven reverse approach in the Reinitz's model, more simplified models are also 
established by assuming a "minimal" set of interactions between genes. For instance, the "two parallel toggle switch" model is based on the strong inhibition between $\mathrm{Hb}$ and $\mathrm{Kni}$, as well as $\mathrm{Gt}$ and $\mathrm{Kr}$ [75]. This model shares the similar ODE equation except that the production term is described with a product of generalized Hill functions:

$$
\begin{aligned}
\frac{\mathrm{d} v_{i}^{a}}{\mathrm{~d} t}= & R_{a} \Pi_{A=1}^{N_{A}} p^{A} \Pi_{R=1}^{N_{R}}\left(1-p^{R}\right) \\
& +D_{a}\left[v_{i-1}^{a}+v_{i+1}^{a}-2 v_{i}^{a}\right]-k_{a} v_{i}^{a}
\end{aligned}
$$

$p^{A}$ and $1-p^{R}$ represents the probability of activation or repression of the other genes to gene a. This model only requires 5-7 parameters, and can simulate major dynamic features of the pattern formation of gap genes in wild type as well as in some mutants. However, none of the current models can capture the dynamics of the gap gene profiles in the whole nc14.

\section{CONCLUSIONS AND OUTLOOK}

Fly embryos have become one of the perfect model systems for quantitative systems biology studies. Compared with the single-cell system, the fly embryo provides a highly reproducible and synchronized multi-cellular system [9]. More importantly, the developmental patterns show single-cell precision and accuracy, and robustness against the environmental fluctuations and genetic variations, yet their formation is highly dynamic and rapid. Hence one of the most interesting and intriguing questions to be addressed in this system is how the regulatory network copes with the gene expression noise during the dynamic development.

The highly dynamic developing fly embryos also impose a great challenge on quantitative measurements of the gene expression patterning. Besides the improvement of the conventional imaging on protein or mRNA profiles, the application of several new imaging methods opens new avenues. The introduction of the MS2-MCP imaging system [76] into fly embryos showed the dynamic transcription activity of several segmentation genes such as $h b$ and eve in live imaging without the maturation effect $[61,77]$. Single molecule FISH (smFISH) allowed counting the mRNA of segmentation genes and revealing the stochastic transcription activity [64]. The combination of immuofluorescence and smFISH allows quantifying the absolute concentration of the transcription factor and the transcription activity in the same embryo, hence it provides an excellent way to measure the transcriptional regulation functions [63]. A digital embryo generated with the $3 \mathrm{D}$ imaging registration method showed the average quantitative spatial-temporal altas of the mRNA distribution in early fly embryos [71]. Furthermore, one would expect that the fast 3D imaging could also be applied to capture the dynamic gene expression in the whole fly embryo with the improvement of the light-sheet microscope $[78,79]$ and adaptive optics [80]. Finally, the optogenetics tools could help perturb the network with an arbitrary spatial-temporal pattern to reveal the elusive interactions between the segmentation genes [81].

Along with the development of the novel quantitative measurement methods, biochemistry and genetics studies will help us to discover new regulation functions of molecules and even identify new regulatory factors in AP pattering gene network. Here are some of the recent examples. Multiple shadow enhancers of the gap genes were found to play a crucial role in generating robust and precise patterning via mediating the dynamic shift and refinement of the gap gene expression [82,83]. And paused pol II was reported to be essential for coordinating pattering via synchronizing the gene expression [84]. A novel F-box protein named fates-shifted (ftd) was reported to play a role in degrading Bcd, which is important in shaping the Bcd gradient [85]. Gene dmpd and sumo were discovered to advance and delay the shutdown of the Bcd dependent transcription of $h b$ at early nc14, respectively $[86,87]$. The endo-siRNA pathway was found to be essential to maintain the robustness of the patterning against the temperature fluctuations. These new discoveries will provide a more comprehensive regulation gene network and may identify the missing key factor, e.g., the posterior gradient predicted in the bi-gradient model [51], which is essential in controlling the noise transmission.

The accumulating quantitative data and new factors in the gene regulation network open new windows for mathematical modeling. The more accurate protein [18] or mRNA profiles [64] measured in the WT and mutant fly lines [35] provide solid training and testing data for searching the parameters describing the gap gene network with a reverse-engineering approach. More importantly, to fully capture the dynamics of the development system, the model needs to emphasize time-dependent transient behaviors, instead of the steady state or a random snapshot. Since the dynamics is associated with temporal-variable inputs (e.g., the dynamics of Bcd gradients $[2,20])$ and the temporal coordinated regulatory switches (e.g., the switch-off of Bcd-dependent $h b$ expression at the beginning of nc14 [86]), it could be better to be accounted by the non-autonomous models with explicit time-dependent parameter changes [86]. On the other hand, mathematical models grounded in physics starts to serve as the "Figure 1 theory" instead of the "Figure 7 theory" in studying biological systems [88], i.e., providing new concepts to direct new testable experiments instead of solely supporting the existing experimental results. For instance, the theoretical prediction that the sharpness of the $\mathrm{Hb}$ boundary is attributed to the non- 
equilibrium transcription opens new experimental frontiers [89].

With the synergy of the quantitative measurements and modeling, we hope we may reveal the underlying mechanism for the embryo developmental system to overcome the ubiquitous gene expression noise and generate highly precise and accurate developmental patterns. This will deepen our understanding of the noise control of the gene network, facilitate searching for more efficient and safer methods for cell programming and reprogramming, and have the great potential for tissue engineering and regenerative medicine.

\section{ACKNOWLEDGEMENTS}

The authors are grateful for the two reviewers for helpful suggestions. We apologize to our colleagues whose work could not be cited due to the page limits. This project is supported by the National Natural Science Foundation of China (No.31670852) and 100-talent plan of Peking University. The Drosophila lab used in this project is supported by Peking-Tsinghua Center for Life Sciences.

\section{COMPLIANCE WITH ETHICS GUIDELINES}

The authors Zhe Yang, Xiaoxuan Wu, Ning Yang and Feng Liu declare that they have no conflict of interests.

This article is a review article and all applicable international, national, and/or institutional guidelines for the care and use of animals were followed.

\section{REFERENCES}

1. Gregor, T., Tank, D. W., Wieschaus, E. F. and Bialek, W. (2007) Probing the limits to positional information. Cell, 130, 153-164

2. Gregor, T., Wieschaus, E. F., McGregor, A. P., Bialek, W. and Tank, D. W. (2007) Stability and nuclear dynamics of the Bicoid morphogen gradient. Cell, 130, 141-152

3. Namba, R., Pazdera, T. M., Cerrone, R. L. and Minden, J. S. (1997) Drosophila embryonic pattern repair: how embryos respond to bicoid dosage alteration. Development, 124, 1393-1403

4. Vincent, A., Blankenship, J. T. and Wieschaus, E. (1997) Integration of the head and trunk segmentation systems controls cephalic furrow formation in Drosophila. Development, 124, 3747-3754

5. Liu, F., Morrison, A. H. and Gregor, T. (2013) Dynamic interpretation of maternal inputs by the Drosophila segmentation gene network. Proc. Natl. Acad. Sci. USA, 110, 6724-6729

6. Eldar, A. and Elowitz, M. B. (2010) Functional roles for noise in genetic circuits. Nature, 467, 167-173

7. Elowitz, M. B., Levine, A. J., Siggia, E. D. and Swain, P. S. (2002) Stochastic gene expression in a single cell. Science, 297, 11831186

8. Ghodsi, Z., Hassani, H. and McGhee, K. (2015) Mathematical approaches in studying bicoid gene. Quant. Biol., 3, 182-192

9. Gregor, T., Garcia, H. G. and Little, S. C. (2014) The embryo as a laboratory: quantifying transcription in Drosophila. Trends Genet.,

\section{$30,364-375$}

10. Rogers, K. W. and Schier, A. F. (2011) Morphogen gradients: from generation to interpretation. Annu. Rev. Cell Dev. Biol., 27, 377407

11. Porcher, A. and Dostatni, N. (2010) The bicoid morphogen system. Curr. Biol., 20, R249-R254

12. Jaeger, J. (2011) The gap gene network. Cell. Mol. Life Sci., 68, 243-274

13. Jaeger, J. (2009) Modelling the Drosophila embryo. Mol. Biosyst., $5,1549-1568$

14. Farrell, J. A. and O'Farrell, P. H. (2014) From egg to gastrula: how the cell cycle is remodeled during the Drosophila mid-blastula transition. Annu. Rev. Genet., 48, 269-294

15. Driever, W. and Nüsslein-Volhard, C. (1988) A gradient of bicoid protein in Drosophila embryos. Cell, 54, 83-93

16. Driever, W. and Nüsslein-Volhard, C. (1988) The bicoid protein determines position in the Drosophila embryo in a concentrationdependent manner. Cell, 54, 95-104

17. Irish, V., Lehmann, R. and Akam, M. (1989) The Drosophila posterior-group gene nanos functions by repressing hunchback activity. Nature, 338, 646-648

18. Dubuis, J. O., Samanta, R. and Gregor, T. (2013) Accurate measurements of dynamics and reproducibility in small genetic networks. Mol. Syst. Biol., 9, 639

19. Surkova, S., Myasnikova, E., Kozlov, K. N., Pisarev, A., Reinitz, J. and Samsonova, M. (2013) Quantitative imaging of gene expression in Drosophila embryos. Cold Spring Harb. Protoc., 2013, 488-497

20. Little, S. C., Tkačik, G., Kneeland, T. B., Wieschaus, E. F. and Gregor, T. (2011) The formation of the Bicoid morphogen gradient requires protein movement from anteriorly localized mRNA. PLoS Biol., 9, e1000596

21. Grimm, O., Coppey, M. and Wieschaus, E. (2010) Modelling the Bicoid gradient. Development, 137, 2253-2264

22. Berezhkovskii, A. M., Sample, C. and Shvartsman, S. Y. (2010) How long does it take to establish a morphogen gradient? Biophys. J., 99, L59-L61

23. Drocco, J. A., Grimm, O., Tank, D. W. and Wieschaus, E. (2011) Measurement and perturbation of morphogen lifetime: effects on gradient shape. Biophys. J., 101, 1807-1815

24. Liu, J., He, F. and Ma, J. (2011) Morphogen gradient formation and action: insights from studying Bicoid protein degradation. Fly (Austin), 5, 242-246

25. Abu-Arish, A., Porcher, A., Czerwonka, A., Dostatni, N. and Fradin, C. (2010) High mobility of bicoid captured by fluorescence correlation spectroscopy: implication for the rapid establishment of its gradient. Biophys. J., 99, L33-L35

26. Liu, J. and Ma, J. (2013) Uncovering a dynamic feature of the transcriptional regulatory network for anterior-posterior patterning in the Drosophila embryo. PLoS One, 8, e62641

27. Margolis, J. S., Borowsky, M. L., Steingrímsson, E., Shim, C. W., Lengyel, J. A. and Posakony, J. W. (1995) Posterior stripe expression of hunchback is driven from two promoters by a common enhancer element. Development, 121, 3067-3077 
28. Lopes, F. J., Vieira, F. M., Holloway, D. M., Bisch, P. M. and Spirov, A. V. (2008) Spatial bistability generates hunchback expression sharpness in the Drosophila embryo. PLoS Comput. Biol., 4, e1000184

29. Hülskamp, M., Lukowitz, W., Beermann, A., Glaser, G. and Tautz, D. (1994) Differential regulation of target genes by different alleles of the segmentation gene hunchback in Drosophila. Genetics, 138, 125-134

30. Schröder, C., Tautz, D., Seifert, E. and Jäckle, H. (1988) Differential regulation of the two transcripts from the Drosophila gap segmentation gene hunchback. EMBO J., 7, 2881-2887

31. Perry, M. W., Bothma, J. P., Luu, R. D. and Levine, M. (2012) Precision of hunchback expression in the Drosophila embryo. Curr. Biol., 22, 2247-2252

32. Surkova, S., Golubkova, E., Manu, L., Panok, L., Mamon, J., Reinitz, M. and Samsonova (2013) Quantitative dynamics and increased variability of segmentation gene expression in the Drosophila Krüppel and knirps mutants. Dev. Biol., 376, 99-112

33. Perry, M. W., Boettiger, A. N. and Levine, M. (2011) Multiple enhancers ensure precision of gap gene-expression patterns in the Drosophila embryo. Proc. Natl. Acad. Sci. USA, 108, 13570 13575

34. Lehmann, R. and Nüsslein-Volhard, C. (1987) hunchback, a gene required for segmentation of an anterior and posterior region of the Drosophila embryo. Dev. Biol., 119, 402-417

35. Petkova, M. D., Tkačik, G., Bialek, W., Wieschaus, E. F. and Gregor, T. (2016) Optimal decoding of information from a genetic network. arXiv: 161208084

36. Petkova, M. D., Little, S. C., Liu, F. and Gregor, T. (2014) Maternal origins of developmental reproducibility. Curr. Biol., 24, 12831288

37. Wolpert, L. (1969) Positional information and the spatial pattern of cellular differentiation. J. Theor. Biol., 25, 1-47

38. He, F., Wen, Y., Deng, J., Lin, X., Lu, L. J., Jiao, R. and Ma, J. (2008) Probing intrinsic properties of a robust morphogen gradient in Drosophila. Dev. Cell, 15, 558-567

39. Houchmandzadeh, B., Wieschaus, E. and Leibler, S. (2002) Establishment of developmental precision and proportions in the early Drosophila embryo. Nature, 415, 798-802

40. Jaeger, J., Surkova, S., Blagov, M., Janssens, H., Kosman, D., Kozlov, K. N., Manu, E., Myasnikova, C. E., Vanario-Alonso, M., Samsonova, M, et al. (2004) Dynamic control of positional information in the early Drosophila embryo. Nature, 430, 368-371

41. Manu, Surkova, S., Spirov, A. V., Gursky, V. V., Janssens, H., Kim, A. R., Radulescu, O., Vanario-Alonso, C. E., Sharp, D. H., Samsonova, M., et al. (2009) Canalization of gene expression in the Drosophila blastoderm by gap gene cross regulation. PLoS Biol., 7, e1000049

42. Green, J. B. and Sharpe, J. (2015) Positional information and reaction-diffusion: two big ideas in developmental biology combine. Development, 142, 1203-1211

43. Turing, A. M. (1952) The chemical basis of morphogenesis. Philosoph. Trans. Royal Soc. London, 237,37-72

44. Gregor, T., Bialek, W., de Ruyter van Steveninck, R. R., Tank, D.
W. and Wieschaus, E. F. (2005) Diffusion and scaling during early embryonic pattern formation. Proc. Natl. Acad. Sci. USA, 102, 18403-18407

45. Grimm, O. and Wieschaus, E. (2010) The Bicoid gradient is shaped independently of nuclei. Development, 137, 2857-2862

46. Cheung, D., Miles, C., Kreitman, M. and Ma, J. (2011) Scaling of the Bicoid morphogen gradient by a volume-dependent production rate. Development, 138, 2741-2749

47. He, F., Wei, C., Wu, H., Cheung, D., Jiao, R. and Ma, J. (2015) Fundamental origins and limits for scaling a maternal morphogen gradient. Nat. Commun., 6, 6679

48. de Lachapelle, A. M. and Bergmann, S. (2010) Precision and scaling in morphogen gradient read-out. Mol. Syst. Biol., 6, 351

49. Bergmann, S., Sandler, O., Sberro, H., Shnider, S., Schejter, E., Shilo, B.-Z. and Barkai, N. (2007) Pre-steady-state decoding of the Bicoid morphogen gradient. PLoS Biol., 5, e46

50. Jaeger, J. (2010) A matter of timing and precision. Mol. Syst. Biol., 6,427

51. Houchmandzadeh, B., Wieschaus, E. and Leibler, S. (2005) Precise domain specification in the developing Drosophila embryo. Phys. Rev. E Stat. Nonlin. Soft Matter Phys., 72, 061920

52. Howard, M. and ten Wolde, P. R. (2005) Finding the center reliably: robust patterns of developmental gene expression. Phys. Rev. Lett., 95, 208103

53. Cheung, D. and Ma, J. (2015) Probing the impact of temperature on molecular events in a developmental system. Sci. Rep., 5, 13124

54. Kuntz, S. G. and Eisen, M. B. (2014) Drosophila embryogenesis scales uniformly across temperature in developmentally diverse species. PLoS Genet., 10, e1004293

55. Lucchetta, E. M., Lee, J. H., Fu, L. A., Patel, N. H. and Ismagilov, R. F. (2005) Dynamics of Drosophila embryonic patterning network perturbed in space and time using microfluidics. Nature, 434, 1134-1138

56. Lucchetta, E. M., Vincent, M. E. and Ismagilov, R. F. (2008) A precise Bicoid gradient is nonessential during cycles 11-13 for precise patterning in the Drosophila blastoderm. PLoS One, 3, e3651

57. Lucchetta, E. M., Carthew, R. W. and Ismagilov, R. F. (2009) The endo-siRNA pathway is essential for robust development of the Drosophila embryo. PLoS One, 4, e7576

58. Crauk, O. and Dostatni, N. (2005) Bicoid determines sharp and precise target gene expression in the Drosophila embryo. Curr. Biol., 15, 1888-1898

59. Kugler, J.-M. and Lasko, P. (2009) Localization, anchoring and translational control of oskar, gurken, bicoid and nanos mRNA during Drosophila oogenesis. Fly (Austin), 3, 15-28

60. Aegerter-Wilmsen, T., Aegerter, C. M. and Bisseling, T. (2005) Model for the robust establishment of precise proportions in the early Drosophila embryo. J. Theor. Biol., 234, 13-19

61. Garcia, H. G., Tikhonov, M., Lin, A. and Gregor, T. (2013) Quantitative imaging of transcription in living Drosophila embryos links polymerase activity to patterning. Curr. Biol., 23, 2140-2145 62. Lucas, T., Ferraro, T., Roelens, B., De Las Heras Chanes, J., 
Walczak, A. M., Coppey, M. and Dostatni, N. (2013) Live imaging of bicoid-dependent transcription in Drosophila embryos. Curr. Biol., 23, 2135-2139

63. Xu, H., Sepúlveda, L. A., Figard, L., Sokac, A. M. and Golding, I. (2015) Combining protein and mRNA quantification to decipher transcriptional regulation. Nat. Methods, 12, 739-742

64. Little, S. C., Tikhonov, M. and Gregor, T. (2013) Precise developmental gene expression arises from globally stochastic transcriptional activity. Cell, 154, 789-800

65. Garcia, H. G., Tikhonov, M., Lin, A. and Gregor, T. (2013) Quantitative imaging of transcription in living Drosophila embryos links polymerase activity to patterning. Curr. Biol., 23, 21402145

66. Golding, I., Paulsson, J., Zawilski, S. M. and Cox, E. C. (2005) Real-time kinetics of gene activity in individual bacteria. Cell, 123, $1025-1036$

67. Reeves, G. T., Trisnadi, N., Truong, T. V., Nahmad, M., Katz, S. and Stathopoulos, A. (2012) Dorsal-ventral gene expression in the Drosophila embryo reflects the dynamics and precision of the dorsal nuclear gradient. Dev. Cell, 22, 544-557

68. Giepmans, B. N., Adams, S. R., Ellisman, M. H., Tsien, R. Y (2006) The fluorescent toolbox for assessing protein location and function. Science, 312, 217-224

69. Myasnikova, E., Samsonova, A., Kozlov, K., Samsonova, M. and Reinitz, J. (2001) Registration of the expression patterns of Drosophila segmentation genes by two independent methods. Bioinformatics, 17, 3-12

70. Blythe, S. A. and Wieschaus, E. F. (2016) Establishment and maintenance of heritable chromatin structure during early Drosophila embryogenesis. eLife, 5, 5

71. Fowlkes, C. C., Hendriks, C. L. L., Keränen, S. V., Weber, G. H., Rübel, O., Huang, M.-Y., Chatoor, S., DePace, A. H., Simirenko, L., Henriquez, C., et al. (2008) A quantitative spatiotemporal atlas of gene expression in the Drosophila blastoderm. Cell, 133, 364374

72. Surkova, S., Kosman, D., Kozlov, K., Manu, E., Myasnikova, A. A., Samsonova, A., Spirov, C. E., Vanario-Alonso, M., Samsonova, J. and Reinitz (2008) Characterization of the Drosophila segment determination morphome. Dev. Biol., 313, 844-862

73. Barrangou, R. (2014) RNA events. Cas 9 targeting and the CRISPR revolution. Science, 344, 707-708

74. Bassett, A. R. and Liu, J.-L. (2014) CRISPR/Cas9 and genome editing in Drosophila. J. Genet. Genomics, 41, 7-19

75. Papatsenko, D. and Levine, M. (2011) The Drosophila gap gene network is composed of two parallel toggle switches. PLoS One, 6 , e21145

76. Bertrand, E., Chartrand, P., Schaefer, M., Shenoy, S. M., Singer, R. H. and Long, R. M. (1998) Localization of ASH1 mRNA particles in living yeast. Mol. Cell, 2, 437-445

77. Bothma, J. P., Garcia, H. G., Esposito, E., Schlissel, G., Gregor, T. and Levine, M. (2014) Dynamic regulation of eve stripe 2 expression reveals transcriptional bursts in living Drosophila embryos. Proc. Natl. Acad. Sci. USA, 111, 10598-10603

78. Keller, P. J. (2013) Imaging morphogenesis: technological advances and biological insights. Science, 340, 1234168

79. Stegmaier, J., Amat, F., Lemon, W. C., McDole, K., Wan, Y., Teodoro, G., Mikut, R. and Keller, P. J. (2016) Real-time threedimensional cell segmentation in large-scale microscopy data of developing embryos. Dev. Cell, 36, 225-240

80. Ji, N., Milkie, D. E. and Betzig, E. (2010) Adaptive optics via pupil segmentation for high-resolution imaging in biological tissues. Nat. Methods, 7, 141-147

81. Huang, A., Amourda, C., Zhang, S., Tolwinski, N. S., and Saunders, T. E. (2017) Decoding temporal interpretation of the morphogen Bicoid in the early Drosophila embryo. eLife, 6, e26258

82. Perry, M. W., Boettiger, A. N., Bothma, J. P. and Levine, M. (2010) Shadow enhancers foster robustness of Drosophila gastrulation. Curr. Biol., 20, 1562-1567

83. El-Sherif, E. and Levine, M. (2016) Shadow enhancers mediate dynamic shifts of gap gene expression in the Drosophila embryo. Curr. Biol., 26, 1164-1169

84. Lagha, M., Bothma, J. P., Esposito, E., Ng, S., Stefanik, L., Tsui, C., Johnston, J., Chen, K., Gilmour, D. S., Zeitlinger, J., et al. (2013) Paused Pol II coordinates tissue morphogenesis in the Drosophila embryo. Cell, 153, 976-987

85. Liu, J. and Ma, J. (2011) Fates-shifted is an F-box protein that targets Bicoid for degradation and regulates developmental fate determination in Drosophila embryos. Nat. Cell Biol., 13, 22-29

86. Liu, J., Xiao, Y., Zhang, T. and Ma, J. (2016) Time to move on: modeling transcription dynamics during an embryonic transition away from maternal control. Fly (Austin), 10, 101-107

87. Liu, J. and Ma, J. (2015) Modulation of temporal dynamics of gene transcription by activator potency in the Drosophila embryo. Development, 142, 3781-3790

88. Phillips, R. (2015) Theory in biology: Figure 1 or Figure 7? Trends Cell Biol., 25, 723-729

89. Estrada, J., Wong, F., DePace, A. and Gunawardena, J. (2016) Information integration and energy expenditure in gene regulation. Cell, 166, 234-244 